Supporting Information

\title{
Selection of insulating elastomers for high- performance intrinsically stretchable transistors
}

Hang Ren, ${ }^{\dagger,}{ }^{\S, \perp}$ Junmo Zhang, ${ }^{\dagger, \perp}$ Yanhong Tong, ${ }^{*}{ }^{\dagger}$ Mingxin Zhang, ${ }^{\dagger}$ Xiaoli Zhao, ${ }^{\dagger}$ Qingxin Tang, ${ }^{*}, \dagger$ and Yichun $\mathrm{Liu}^{\dagger}$

${ }^{\dagger}$ Centre for Advanced Optoelectronic Functional Materials Research and Key Laboratory of UV-Emitting Materials and Technology, Ministry of Education, Northeast Normal University, Changchun 130024, P. R. China

${ }^{\S}$ Key Laboratory of Opto-electronic Measurement and Optical Information Transmission Technology, Ministry of Education, Changchun University of Science and Technology, Changchun 130022, P. R. China

*E-mail address: tangqx@nenu.edu.cn; tongyh@nenu.edu.cn

KEYWORDS: elastomers, soft materials, stretchable electronics, flexible electronics, organic field-effect transistors 
Table S1. Field-effect characteristics of fabricated DNTT OFETs with different insulating elastomers.

\begin{tabular}{|c|c|c|c|c|}
\hline Dielectric & $\begin{array}{c}\text { Mobility }(\mu)\left(\mathrm{cm}^{2} \mathrm{~V}^{-1} \mathrm{~s}^{-1}\right) \\
\text { (highest) avg. } \pm \text { avg. } \\
\text { dev }\end{array}$ & $\begin{array}{c}\text { On-off ratio } \\
\left(I_{o n} / I_{o f f}\right) \\
\text { avg. }\end{array}$ & $\begin{array}{c}\text { Threshold voltage } \\
\left(V_{T}\right)(V) \text { avg. } \pm \\
\text { avg. dev }\end{array}$ & $\begin{array}{l}\text { Subthreshold swing } \\
\text { (S.S.) (V/decade) avg. } \pm \\
\text { avg. dev }\end{array}$ \\
\hline SEBS H1221 & - & - & - & - \\
\hline SEBS H1052 & $0.04) 0.04 \pm 0.02$ & $5.64 \times 10^{5}$ & $5.82 \pm 0.87$ & $1.80 \pm 0.42$ \\
\hline DC 3-1965 & $0.83 \pm 0.19$ & $8.36 \times 10^{5}$ & $29.1 \pm 7.66$ & $1.55 \pm 0.33$ \\
\hline Sylgard-184 & $(1.42) 1.17 \pm 0.11$ & $8.66 \times 10^{6}$ & $17.39 \pm 4.33$ & $1.28 \pm 0.16$ \\
\hline Sylgard-186 & (1.46) $1.18 \pm 0.13$ & $8.92 \times 10^{6}$ & $15.39 \pm 5.33$ & $0.99 \pm 0.38$ \\
\hline
\end{tabular}


Table S2. Summary of the reported representative intrinsically stretchable transistors.

\begin{tabular}{|c|c|c|c|}
\hline $\begin{array}{c}\text { Source \& drain/gate/channel/dielectric } \\
\text { materials/substrate }\end{array}$ & $\begin{array}{c}\text { Strain } \\
(\%)\end{array}$ & $\begin{array}{c}\mu_{1} / \mu_{2}^{*} \\
\left(\mathrm{~cm}^{2} V^{-1} \mathrm{~s}^{-1}\right)\end{array}$ & Ref. \\
\hline $\begin{array}{l}\text { CNTs,Au/CNTs,Au/P3HT NW, Sylgard-184/ } \\
\text { cross-linked CTBN /Sylgard-184 }\end{array}$ & 34 & $0.61 / 0.08$ & 1 \\
\hline $\begin{array}{c}\text { CNTs/CNTs/DPP-based polymer/ Sylgard-184/ } \\
\text { Sylgard-184 }\end{array}$ & 100 & $1.27 / 0.1$ & 2 \\
\hline $\begin{array}{c}\text { AuNPs-AgNWs, PDMS/AuNPs-AgNWs, PDMS/ } \\
\text { m-CNT-doped P3HT-NFs, Sylgard-184/ion gel/ } \\
\text { Sylgard-184 }\end{array}$ & 50 & $7.38 / 2.44$ & 3 \\
\hline MWCNTs/MWCNTs/SWCNTs/ion gel/Sylgard-184 & 100 & $13.5 / 5$ & 4 \\
\hline $\begin{array}{c}\text { CNTs/CNTs/DPPTT/Sylgard-184/Sylgard-184/ } \\
\text { Sylgard-184 }\end{array}$ & 100 & $0.4 / 5 \times 10^{-4}$ & 5 \\
\hline $\begin{array}{c}\text { CNTs/CNTs/IDTBT/Sylgard-184/Sylgard-184/ } \\
\text { Sylgard-184 }\end{array}$ & 100 & $1.8 / 0.6$ & 5 \\
\hline $\begin{array}{c}\text { AuNWs- Sylgard-184/AuNWs- Sylgard-184/P3HT/ } \\
\text { ion gel/ Sylgard-184 }\end{array}$ & 100 & $-/-$ & 6 \\
\hline CNTs/CNTs/SWCNTs/SEBS/Sylgard-184 & 50 & $10.45 / 2.01$ & 7 \\
\hline $\begin{array}{c}\text { PEDOT:PSS-CNT/CNT/DPP-polymer/ } \\
\text { Sylgard-184/SEBS }\end{array}$ & 100 & $0.34 / 0.1$ & 8 \\
\hline CNTs/E GaIn/P3/FeCl 2 -PDMS/SEBS H1062 & 100 & $0.22 / 0.08$ & 9 \\
\hline CNTs/CNTs/DPPT-TT blend/SEBS H1052/SEBS H1221 & 100 & $0.59 / 0.55$ & 10 \\
\hline $\begin{array}{c}\text { CNTs/CNTs/CONPHINE/crosslinking SEBS H1052/ } \\
\text { SEBS H } 1221\end{array}$ & 100 & $1.78 / 0.99$ & 11 \\
\hline CNTs/CNTs/C12-DDP/SEBS H1062/SEBS H1221 & 100 & $0.463 / \sim 0.1$ & 12 \\
\hline EGaIn/CNTs/P3HT/PU/PU & 160 & $0.034 /-$ & 13 \\
\hline AgNWs/AgNWs/SWCNTs/CN990:SR550:SR259/PUA & 50 & $32.5 / 11.7(20 \%)$ & 14 \\
\hline CNTs/CNTs/Semiconducting CNTs/TPU/TPU & 100 & $0.18 / \sim 0.11$ & 15 \\
\hline $\begin{array}{l}\text { AgNWs/PEDOT:PSS, PU/PSe-DPP/PII-2T/ } \\
\text { PVDF-HFP\&PVP/SEBS (Kraton-D1102A) }\end{array}$ & 80 & $0.199 / 0.01$ & 16 \\
\hline $\begin{array}{l}\text { CNTs/CNTs/Semiconducting CNTs/PUU12/ } \\
\text { styrene-isoprene copolymer }\end{array}$ & 50 & $\sim 10 / \sim 4$ & 17 \\
\hline PEDOT:PSS /PEDOT:PSS/ PTB7-Th /iTPU/iTPU & 80 & $17.8 / 12.2(20 \%)$ & 18 \\
\hline $\begin{array}{c}\text { PEDOT:PSS-CNT/PEDOT:PSS-CNT/PIDTBT/ } \\
\text { Sylgard-186/PDMS }\end{array}$ & 100 & $1.78 / 1.16$ & This work \\
\hline
\end{tabular}


Table S3. Change of the channel dimension and dielectric capacitance under different strain perpendicular and parallel to channel length direction.

\begin{tabular}{|c|c|c|c|c|c|c|c|}
\hline \multicolumn{2}{|c|}{ Strain $(\%)$} & 0 & 20 & 40 & 60 & 80 & 100 \\
\hline \multirow{2}{*}{$L / W$} & $+\| \rightarrow$ & 0.1 & 0.128 & 0.162 & 0.191 & 0.214 & 0.246 \\
\hline & $\Leftrightarrow$ & 0.1 & 0.082 & 0.066 & 0.056 & 0.048 & 0.041 \\
\hline \multicolumn{2}{|c|}{$C_{\mathrm{i}}\left(\mathrm{nF} \mathrm{cm}^{-2}\right)$} & 1.60 & 1.71 & 1.78 & 1.87 & 1.97 & 2.07 \\
\hline
\end{tabular}




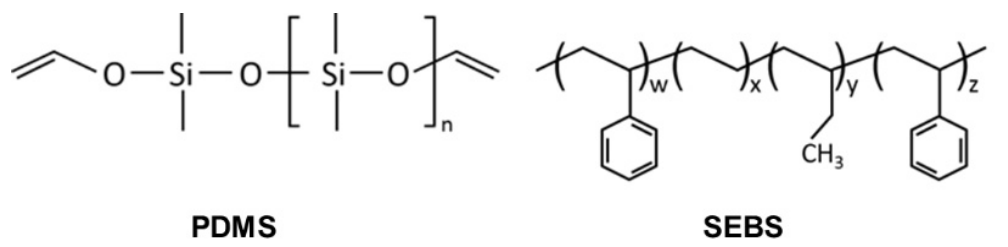

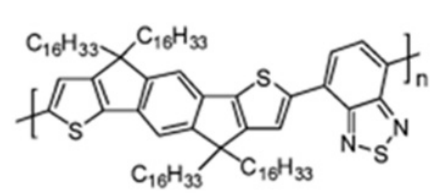

PIDTBT

Figure S1. Chemical structure of PDMS, SEBS and PIDTBT, respectively. For SEBS H1221 and $\mathrm{H} 1052, \mathrm{x}+\mathrm{y}$ value is $88 \%$ and $80 \%$, respectively.

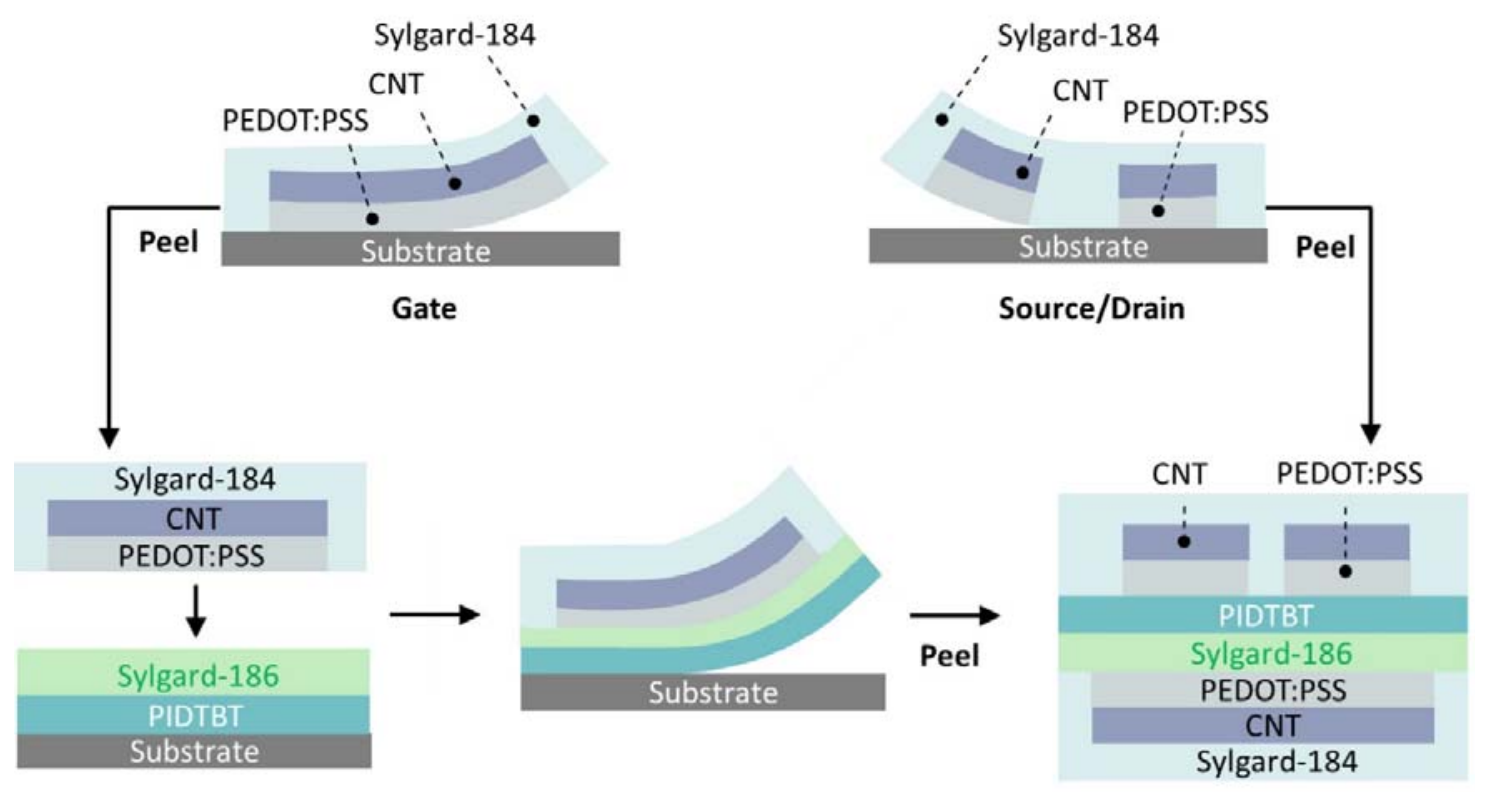

Figure S2. Schematic illustration of the fabrication process for the intrinsically stretchable OFETs. 

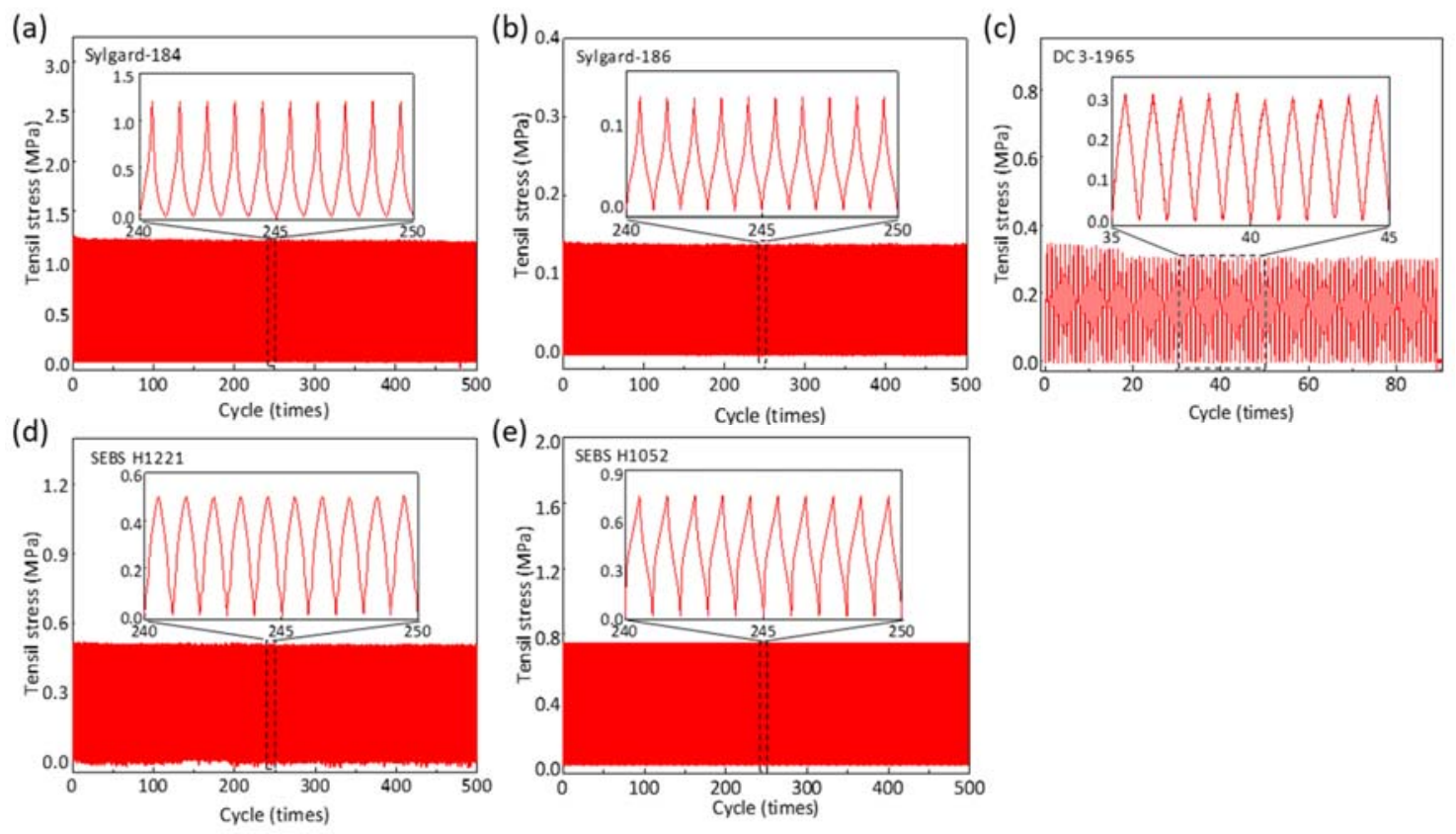

Figure S3. Cyclic tensile tests of insulating elastomers with a strain of $70 \%$. The insets show the zoomed-in plot of the middle part.
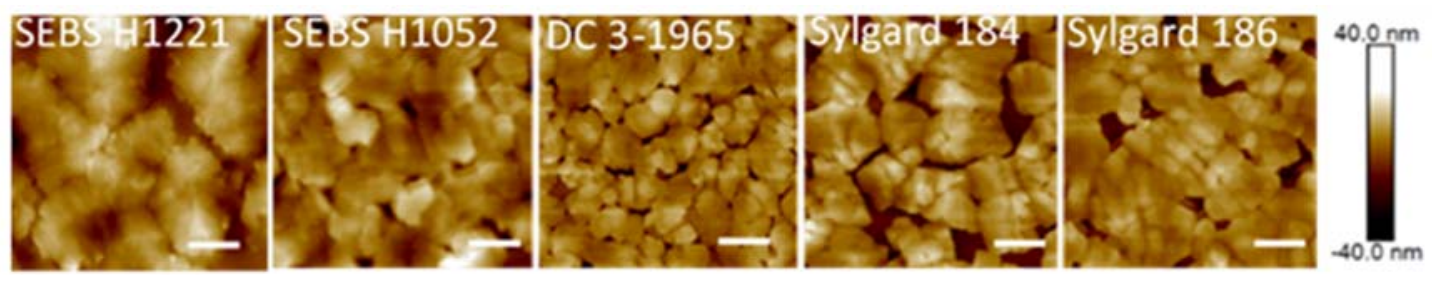

FigureS4. AFM morphology of the DNTT films on different dielectrics (scale bar: $1 \mu \mathrm{m}$ ). 


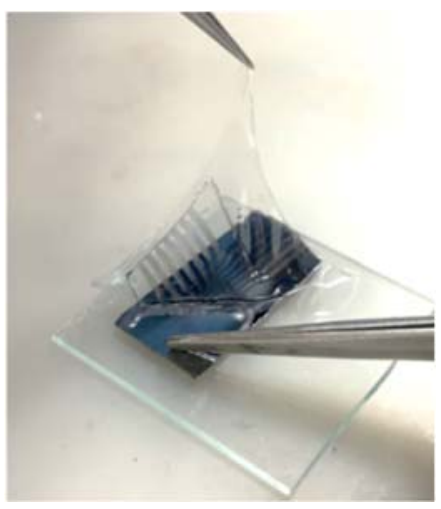

Figure S5. Peeling process of the PEDOT:PSS/CNT electrodes embedded in SEBS.
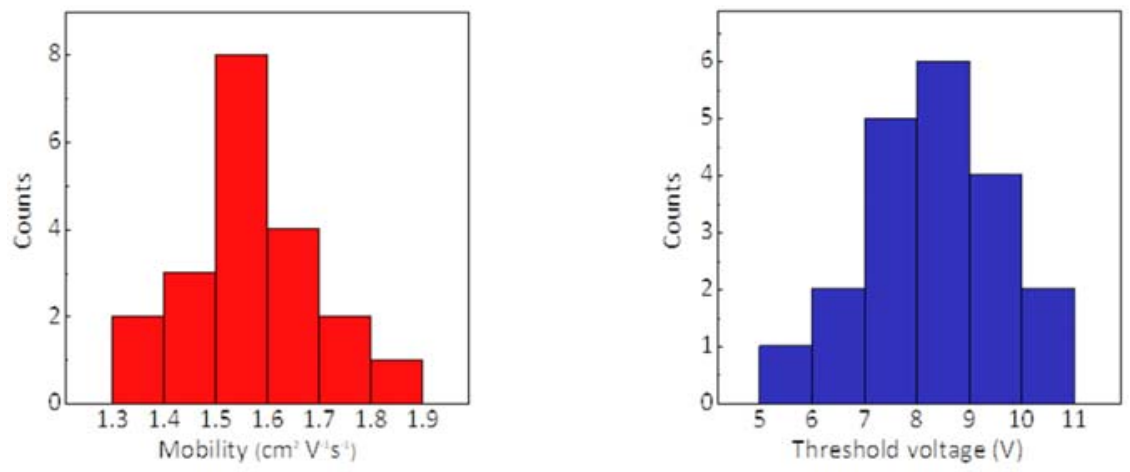

Figure S6. Histogram distributions of field-effect performance of the intrinsically stretchable transistors (20 devices).
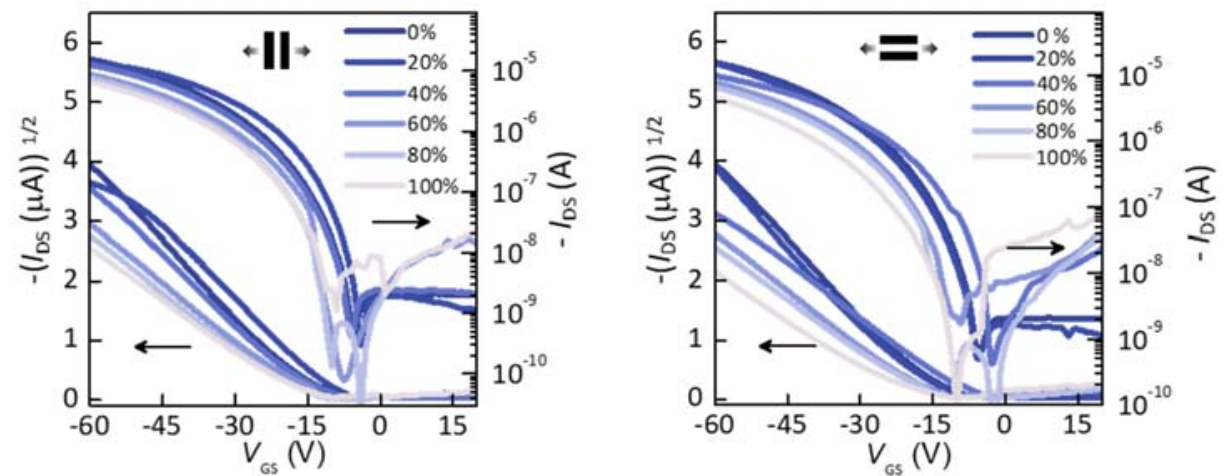

Figure S7. Transfer curves of the intrinsically stretchable OFETs under different strains parallel and perpendicular to the channel length direction, respectively. 


\section{References}

(1) Kang, B.; Song, E.; Lee, S. B.; Chae, B.-G.; Ahn, H.; Cho, K., Stretchable Polymer Gate Dielectric with Segmented Elastomeric Network for Organic Soft Electronics. Chem. Mater. 2018, 30 (18), 6353-6360.

(2) Wang, G.-J. N.; Zheng, Y.; Zhang, S.; Kang, J.; Wu, H.-C.; Gasperini, A.; Zhang, H.; Gu, X.; Bao, Z., Tuning the Cross-Linker Crystallinity of a Stretchable Polymer Semiconductor. Chem. Mater. 2019, 31 (17), 6465-6475.

(3) Sim, K.; Rao, Z.; Kim, H.-J.; Thukral, A.; Shim, H.; Yu, C., Fully rubbery integrated electronics from high effective mobility intrinsically stretchable semiconductors. Sci. Adv. 2019, 5 (2), eaav5749.

(4) Wu, M.-Y.; Wang, J.; Arnold, M. S., Channel length scaling of over $100 \%$ biaxially stretchable carbon nanotube transistors. Appl. Phys. Lett. 2019, 114 (14), 143301.

(5) Zheng, Y.; Wang, G.-J. N.; Kang, J.; Nikolka, M.; Wu, H.-C.; Tran, H.; Zhang, S.; Yan, H.; Chen, H.; Yuen, P. Y.; Mun, J.; Dauskardt, R. H.; McCulloch, I.; Tok, J. B.-H.; Gu, X.; Bao, Z., An Intrinsically Stretchable High-Performance Polymer Semiconductor with Low Crystallinity. Adv. Funct. Mater. 2019, 29 (46), 1905340.

(6) Zhu, B.; Gong, S.; Lin, F.; Wang, Y.; Ling, Y.; An, T.; Cheng, W., Patterning Vertically Grown Gold Nanowire Electrodes for Intrinsically Stretchable Organic Transistors. Adv. Electron. Mater. 2019, 5 (1), 1800509.

(7) Jiao, H.; Zhang, M.; Du, C.; Zhang, Z.; Huang, W.; Huang, Q., Intrinsically stretchable allcarbon-nanotube transistors with styrene-ethylene-butylene-styrene as gate dielectrics integrated by photolithography-based process. RSC Adv. 2020, 10 (14), 8080-8086.

(8) Oh, J. Y.; Rondeau-Gagne, S.; Chiu, Y.-C.; Chortos, A.; Lissel, F.; Wang, G.-J. N.; Schroeder, B. C.; Kurosawa, T.; Lopez, J.; Katsumata, T.; Xu, J.; Zhu, C.; Gu, X.; Bae, W.-G.; Kim, Y.; Jin, L.; Chung, J. W.; Tok, J. B. H.; Bao, Z., Intrinsically stretchable and healable semiconducting polymer for organic transistors. Nature 2016, 539 (7629), 411 415.

(9) Rao, Y.-L.; Chortos, A.; Pfattner, R.; Lissel, F.; Chiu, Y.-C.; Feig, V.; Xu, J.; Kurosawa, T.; Gu, X.; Wang, C.; He, M.; Chung, J. W.; Bao, Z., Stretchable Self-Healing Polymeric Dielectrics Cross-Linked Through Metal-Ligand Coordination. J. Am. Chem. Soc. 2016, 138 (18), 6020-6027.

(10)Xu, J.; Wang, S.; Wang, G.-J. N.; Zhu, C.; Luo, S.; Jin, L.; Gu, X.; Chen, S.; Feig, V. R.; To, J. W. F.; Rondeau-Gagne, S.; Park, J.; Schroeder, B. C.; Lu, C.; Oh, J. Y.; Wang, Y.; Kim, Y.-H.; Yan, H.; Sinclair, R.; Zhou, D.; Xue, G.; Murmann, B.; Linder, C.; Cai, W.; Tok, J. B. H.; Chung, J. W.; Bao, Z., Highly stretchable polymer semiconductor films through the nanoconfinement effect. Science 2017, 355 (6320), 59.

(11)Wang, S.; Xu, J.; Wang, W.; Wang, G.-J. N.; Rastak, R.; Molina-Lopez, F.; Chung, J. W.; Niu, S.; Feig, V. R.; Lopez, J.; Lei, T.; Kwon, S.-K.; Kim, Y.; Foudeh, A. M.; Ehrlich, A.; Gasperini, A.; Yun, Y.; Murmann, B.; Tok, J. B. H.; Bao, Z., Skin electronics from scalable fabrication of an intrinsically stretchable transistor array. Nature 2018, 555 (7694), 83. 
(12)Mun, J.; Wang, G.-J. N.; Oh, J. Y.; Katsumata, T.; Lee, F. L.; Kang, J.; Wu, H.-C.; Lissel, F.; Rondeau-Gagné, S.; Tok, J. B.-H.; Bao, Z., Effect of Nonconjugated Spacers on Mechanical Properties of Semiconducting Polymers for Stretchable Transistors. Adv. Funct. Mater. 2018, 28 (43), 1804222.

(13)Chortos, A.; Lim, J.; To, J. W. F.; Vosgueritchian, M.; Dusseault, T. J.; Kim, T.-H.; Hwang, S.; Bao, Z., Highly Stretchable Transistors Using a Microcracked Organic Semiconductor. Adv. Mater. 2014, 26 (25), 4253-4259.

(14)Liang, J.; Li, L.; Chen, D.; Hajagos, T.; Ren, Z.; Chou, S.-Y.; Hu, W.; Pei, Q., Intrinsically stretchable and transparent thin-film transistors based on printable silver nanowires, carbon nanotubes and an elastomeric dielectric. Nat. Commun. 2015, 6 (1), 7647.

(15)Chortos, A.; Koleilat, G. I.; Pfattner, R.; Kong, D.; Lin, P.; Nur, R.; Lei, T.; Wang, H.; Liu, N.; Lai, Y.-C.; Kim, M.-G.; Chung, J. W.; Lee, S.; Bao, Z., Mechanically Durable and Highly Stretchable Transistors Employing Carbon Nanotube Semiconductor and Electrodes. Adv. Mater. 2016, 28 (22), 4441-4448.

(16)Lu, C.; Lee, W.-Y.; Shih, C.-C.; Wen, M.-Y.; Chen, W.-C., Stretchable Polymer Dielectrics for Low-Voltage-Driven Field-Effect Transistors. ACS Appl. Mater. Interfaces 2017, 9 (30), 25522-25532.

(17)Khatib, M.; Huynh, T.-P.; Deng, Y.; Horev, Y. D.; Saliba, W.; Wu, W.; Haick, H., A Freestanding Stretchable and Multifunctional Transistor with Intrinsic Self-Healing Properties of all Device Components. Small 2019, 15 (2), 1803939.

(18)Park, D. H.; Park, H. W.; Chung, J. W.; Nam, K.; Choi, S.; Chung, Y. S.; Hwang, H.; Kim, B.; Kim, D. H., Highly Stretchable, High-Mobility, Free-Standing All-Organic Transistors Modulated by Solid-State Elastomer Electrolytes. Adv. Funct. Mater. 2019, 29 (18), 1808909. 